\title{
Nuclear Spin Relaxation
}

\author{
Friend and Foe
}

\section{Malcolm H Levitt}

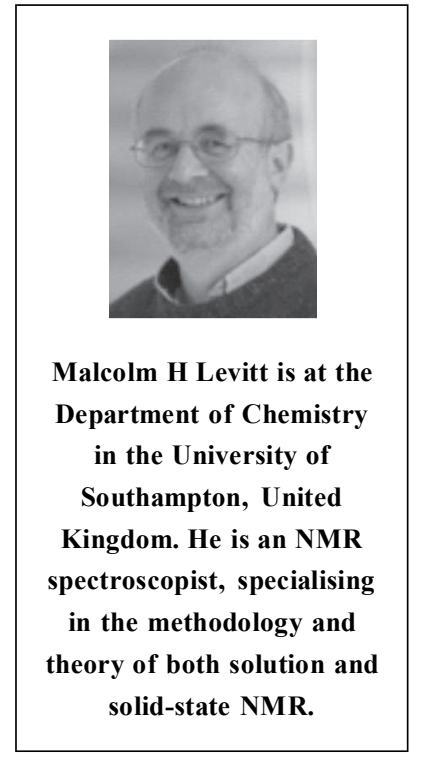

\section{Keywords}

Nuclear magnetic resonance, relaxation, long-lived states.
This article attempts to explain what nuclear relaxation is, why it can be a friend, why it can also be a foe, and how the destructive qualities of relaxation may sometimes be held at bay using the symmetry properties of the quantum states and of the relaxation processes.

\section{Introduction}

In the context of nuclear magnetic resonance (NMR), the term relaxation indicates the process by which the magnetic atomic nuclei reach thermal equilibrium with the chaotic molecular environment. In NMR, this process can be very slow, requiring between a fraction of a second to many minutes, depending on the sample and the environmental conditions. To NMR spectroscopists, relaxation is a friend, since it generates the nuclear magnetisation from which all NMR signals are derived; at the same time, relaxation can be a foe, since it limits how long any different forms of spin order last. For many years, it was generally thought that relaxation was intrinsic to the sample, and could not readily be manipulated or overcome. However, recent experiments have shown that in some cases the nuclear spin systems may be held in special configurations called long-lived states that are to some extent protected against relaxation.

\section{Spin-Lattice Relaxation: Our Friend}

Matter is made of molecules, molecules are made of atoms, and inside every atom is a nucleus. Nuclei occur in many different species, called nuclides, which are defined by the numbers of protons and neutrons they contain. The chemical nature of an atom is defined by the number of protons in its nucleus. For example, all atoms of carbon have six protons in the nucleus, and all atoms of hydrogen have a single proton in the nucleus. 
Isotopes of an element contain atoms whose nuclei have the same number of protons but different numbers of neutrons. For example, all hydrogen nuclei have a single proton, while all carbon nuclei have six protons. The most common isotope of hydrogen is denoted ${ }^{1} \mathrm{H}$, indicating that the nucleus has a single proton and no neutron. Nuclei of the rare hydrogen isotope called deuterium (or heavy hydrogen), denoted ${ }^{2} \mathrm{H}$, contain one proton and one neutron. The most common nucleus of carbon has six protons and six neutrons and is denoted ${ }^{12} \mathrm{C}$ (pronounced 'carbon-12'). About $1 \%$ of all carbon atoms have a nucleus containing six protons and seven neutrons. Carbon atoms with such nuclei are denoted ${ }^{13} \mathrm{C}$ (pronounced 'carbon-13').

Some nuclides are magnetic and have a magnetic moment, which means that they interact with applied magnetic fields. For example, the nuclei of ${ }^{1} \mathrm{H}$ and ${ }^{13} \mathrm{C}$ are both magnetic, although ${ }^{1} \mathrm{H}$ is more magnetic than ${ }^{13} \mathrm{C}$. The common ${ }^{12} \mathrm{C}$ isotope of carbon is not magnetic. Nuclear magnetic resonance (NMR) detects the bulk magnetism of the nuclei, i.e., the sum of all the microscopic magnetic moments in the sample. NMR signals may therefore be obtained from the nuclei of ${ }^{1} \mathrm{H}$ and ${ }^{13} \mathrm{C}$, but not from ${ }^{12} \mathrm{C}$ nuclei.

An ordinary cup of water, lying on a table outside a strong magnetic field, contains about $10^{25}$ magnetic proton nuclei. However, the orientations of the proton magnetic moments are completely random, pointing in every possible direction, so the net magnetic moment from the collection of nuclei cancels out, to a good approximation [1]. For every proton nucleus with a magnetic moment pointing in one direction, there will be another one pointing in the opposite direction, so that the net moment cancels out. As a result, bulk nuclear magnetism is very weak and hard to observe.

Suppose now that the water is placed in a very strong magnetic field. The magnetic moment of each ${ }^{1} \mathrm{H}$ nucleus interacts with the magnetic field. The energy of the magnetic nucleus is slightly lower when aligned in the same direction to the field, compared to when it is aligned in the opposite direction to the field. The same
Many common atomic nuclei are weakly magnetic, leading to the phenomenon of nuclear magnetism. 
Figure 1. (a) The magnetic moments of the nuclei are randomly oriented outside the magnetic field. (b) When the sample is placed in the magnetic field, a small net alignment of the nuclei along the field is developed (greatly exaggerated in the picture). The time taken for this bulk magnetic moment to develop is called the spin-lattice relaxation time, $T_{1}$.

The net alignment of nuclei along a magnetic field is very weak, since nuclear magnetic energies are much smaller than thermal energy (unlike the case of a compass needle).

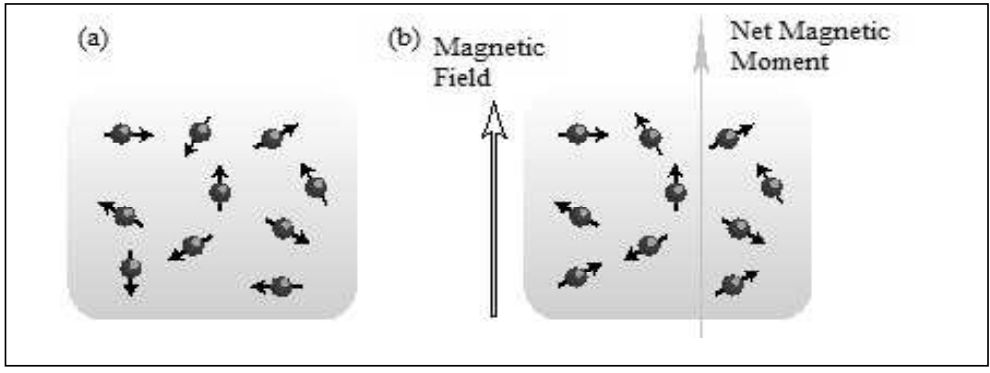

principle applies to a compass needle: a compass needle aligns with an external magnetic field in order to minimize the magnetic energy. Similarly, the magnetic nuclei in a sample of water align, to a very small extent, along the external magnetic field. This magnetic alignment is only slight, but it exists. A small bulk magnetic moment arises in the sample, generated by the slight net alignment of the magnetic nuclei along the applied magnetic field. The process is depicted in Figure 1.

Although very small, this bulk magnetic moment is essential for NMR spectroscopy. All the numerous NMR experiments which allow the determination of molecular structure, molecular motion, and anatomical structure, rely on the very small bulk magnetic moment, which develops when a sample is exposed to a strong magnetic field.

The bulk nuclear magnetism does not develop instantaneously. It takes some time. It develops through the weak interactions of the nuclear magnetic moments with the tumbling motions of the molecules in solution. In the jargon of NMR, the development of the bulk magnetic moment along an applied magnetic field is due to a process called spin-lattice relaxation. It is characterized by a time constant called the spin-lattice relaxation time constant, and which is usually denoted $T_{1}$. For the ${ }^{1} \mathrm{H}$ nuclei of water, $T_{1}$ is typically around 5 seconds.

The net alignment of nuclear magnetic moments with an applied magnetic field is very slight. Out of every 100,000 nuclei, only one more nuclear moment will be aligned (on average) along the field than against it. This alignment effect is so small because 
nuclear magnetic energies are minute. In particular, nuclear magnetic energies are much smaller than the thermal energy $k_{\mathrm{B}} T$, where $k_{\mathrm{B}}$ is the Boltzmann constant, and $T$ is the absolute temperature (in degrees Kelvin). Hence, under ordinary conditions, the randomizing effect of thermal motion is far larger than the magnetic energy of nuclei. The nuclear magnetism is scrambled and almost completely destroyed by the chaotic thermal environment. Fortunately, the havoc wreaked by thermal molecular motion is not complete. The tiny residual nuclear magnetism (of the order of $10^{-5}$ ), which is established through spin-lattice relaxation, is the basis of almost all NMR and MRI experiments $[1]$.

\section{Spin-Lattice Relaxation: Our Foe}

If magnetic nuclei could be completely aligned along the magnetic field, the bulk nuclear magnetism, and hence the strength of NMR signals, would be enormously increased. Recently, a raft of experimental hyperpolarization methods have been developed, which generate materials in states exhibiting substantial degrees of nuclear alignment, and hence enormously enhanced NMR signals. In some cases, NMR signal enhancements by factors of the order of 10,000 have been observed experimentally. A discussion of these methods is beyond the scope of this article: the techniques are complex and often involve combinations of very low temperatures, strong magnetic fields, as well as microwave or laser irradiation. Despite their technical complexity, some of these methods have been commercialized, and used for clinical trials, in which hyperpolarized NMR is used to characterize tumours in human cancer patients. Such experiments would be impossible without the large gain in signal strength provided by hyperpolarization.

Unfortunately, the hyperpolarized state has a limited lifetime. Spin-lattice relaxation gradually destroys the nuclear hyperpolarization, bringing the sample back into equilibrium, so that the bulk nuclear magnetization returns to its tiny thermal equilibrium value (Figure 2). This process occurs over a
Materials may be prepared in hyperpolarized states, providing greatly enhanced NMR signals. 
Figure 2. (a) A hyperpolarized material exhibits a greatly increased net alignment of nuclear magnetic moments along a magnetic field.

(b) The hyperpolarization decays with time constant $T_{1}$, leading to the re-establishment of the thermal equilibrium state with a small net magnetic moment.

Figure 3. A molecule of DBT (2,3-dibromothiophene) contains two hydrogen atoms, which have magnetic nuclei (denoted by the arrows).

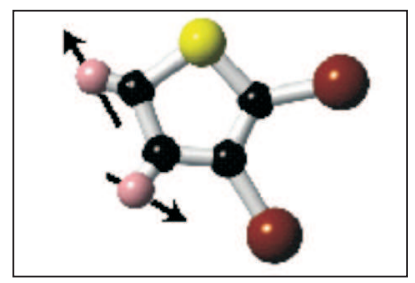

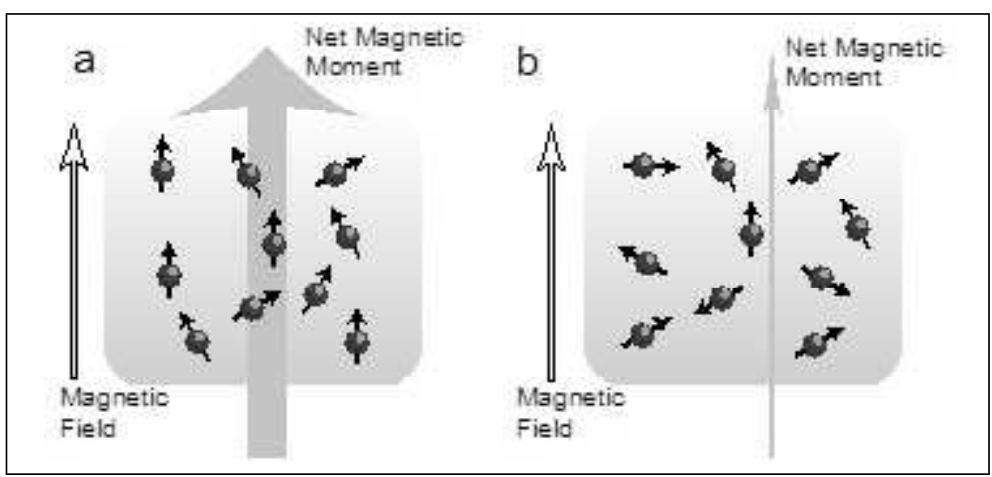

timescale defined by the spin-lattice relaxation time constant $T_{1}$. So whereas spin-lattice relaxation is the friend of the NMR spectroscopist, since it allows bulk nuclear magnetism to develop in a strong magnetic field, it can also be the foe of the spectroscopist, since it leads to the limited lifetime of hyperpolarized states, which are capable of generating much larger NMR signals.

\section{Dipole-Dipole Relaxation}

Relaxation occurs because atomic nuclei interact with the thermal molecular surroundings. But how does this work, in detail?

Nuclear relaxation can be a very complex affair [2]. However, in favourable cases, a simplified qualitative treatment conveys the main principles.

One such case arises when the molecules contain only two magnetic nuclei. An example is a molecule of DBT (2,3dibromothiophene), as shown in Figure 3. This molecule contains four carbon atoms, two bromine atoms, a sulphur atom, and two hydrogen atoms. The most common isotopes of carbon and sulphur have non-magnetic nuclei; although bromine nuclei are magnetic, it turns out that they may ignored in the present context, for technical reasons too complex to explain here. So consider, for simplicity, that DBT only contains two magnetic nuclei, which are both protons (nuclei of ${ }^{1} \mathrm{H}$ ).

Each hydrogen nucleus of DBT generates a small magnetic field which loops around in space, just like that generated by a bar 

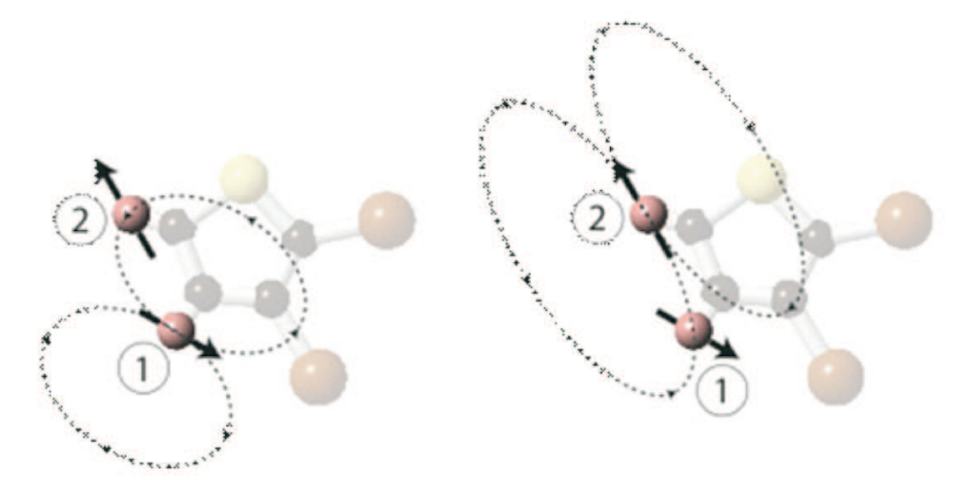

magnet, and is sensed by the other hydrogen nucleus (Figure 4). This magnetic interaction is called the magnetic dipole-dipole coupling. The magnitude of this coupling is proportional to the inverse third power of the distance between the nuclei $[1,2]$.

As shown in Figure 4, the magnitude and direction of the magnetic field that one nucleus generates at the site of the other nucleus depends on two independent factors: (1) the orientation of the nuclear magnetic moment that generates the local field and (2) the orientation of the DBT molecule in space. Hence, as the DBT molecules rotate in solution, the direction and magnitude of the local magnetic fields change. The thermally induced tumbling of DBT molecules in solution thereby generates rapidly fluctuating local magnetic fields, that couple to the magnetic nuclei. This comprises the dipole-dipole mechanism of nuclear relaxation. In DBT, this is the major cause of spin-lattice relaxation. There are other mechanisms too, but this is the most important one.

A detailed analysis shows that the relaxation rate constants generated by the dipole-dipole mechanism are proportional to the square of the dipole-dipole coupling, and hence are proportional to the inverse sixth power of the distance between the nuclei [2]. Experimental estimates of such rates therefore lead to estimates of distance between the magnetic nuclei, and hence to constraints on the molecular geometry. This is one of the major ways to estimate the molecular structure by NMR spectroscopy - although this topic is beyond the scope of this article.
Figure 4. In DBT, hydrogen nucleus 1 generates a magnetic field that is sensed by nucleus 2 (left). At the same time, nucleus 2 generates a magnetic field that is sensed by nucleus 1 (right).
The mutual magnetic dipole-dipole coupling of two nuclei is symmetric with respect to nuclear exchange. This means that swapping the two nuclei makes no difference to the coupling. 
For a pair of nuclei, the triplet states are exchange-symmetric, while the singlet state is exchangeantisymmetric. This means that the singlet state changes sign when the nuclei are exchanged.
A key feature of the magnetic dipole-dipole coupling between two nuclei is that it is exchange-symmetric: if the positions of the two nuclei are exchanged, then the coupling remains the same.

\section{Exchange Symmetry}

The hydrogen nuclei of water are both protons, which belong to a class of particles with spin-1/2. A detailed explanation of this term is beyond the scope of this article, but in essence it means that the quantum state of each proton may always be described using just two quantum states, usually denoted $|\alpha\rangle$ and $|\beta\rangle$, which possess angular momentum $\pm 1 / 2 \hbar$ along some external direction (often chosen to be the direction of the applied magnetic field, although this choice is not essential). The symbol $\hbar$ (pronounced 'h-bar') denotes the Planck constant divided by $2 \pi$, where the Planck constant is one of the fundamental constants of Nature, and which plays a central role in quantum theory.

Since each proton may be described using two states, there are four possible quantum states of a proton pair, which may be denoted $\left|\alpha_{1} \alpha_{2}\right\rangle,\left|\alpha_{1} \beta_{2}\right\rangle,\left|\beta_{1} \alpha_{2}\right\rangle$ and $\left|\beta_{1} \beta_{2}\right\rangle$. Any mixture of these states is also a valid state for the proton pair, so (for example), the state $\left|\alpha_{1} \beta_{2}\right\rangle+\left|\beta_{1} \alpha_{2}\right\rangle$ is also a valid quantum state (some numerical factors have been omitted, for the sake of simplicity).

Consider now the effect of exchanging the nuclei. The exchange operation may be denoted $\hat{X}$, where the 'hat' denotes a quantummechanical operator. The following equation shows what happens when the exchange operator is applied to one of the quantum states: $\hat{X}\left|\alpha_{1} \beta_{2}\right\rangle=\left|\beta_{1} \alpha_{2}\right\rangle$. Applying the operator to the state, exchanges the labels of the two protons.

The following four states have special properties under the exchange operation:

$$
\begin{aligned}
\left|T_{+1}\right\rangle & =\left|\alpha_{1} \alpha_{2}\right\rangle, \\
\left|T_{+1}\right\rangle & =\left|\alpha_{1} \beta_{2}\right\rangle+\left|\beta_{1} \alpha_{2}\right\rangle \quad|S\rangle=\left|\alpha_{1} \beta_{2}\right\rangle-\left|\beta_{1} \alpha_{2}\right\rangle, \\
\left|T_{-1}\right\rangle & =\left|\beta_{1} \beta_{2}\right\rangle .
\end{aligned}
$$


The three states on the left are called triplet states. They are symmetric under exchange, meaning that exchanging the two nuclei leaves the state unchanged. The one on the right-hand side is called the singlet state, and it has a different symmetry [3]. It is antisymmetric under exchange, which means that the exchange operation changes the sign of the quantum state:

$$
\begin{aligned}
& \hat{X}\left|T_{+1}\right\rangle=\left|T_{+1}\right\rangle, \\
& \hat{X}\left|T_{0}\right\rangle=\left|T_{0}\right\rangle \quad \hat{X}|\mathrm{~S}\rangle=-|S\rangle \\
& \hat{X}\left|T_{-1}\right\rangle=\left|T_{-1}\right\rangle
\end{aligned}
$$

So, for the two-proton system, it is possible to construct 3 quantum states that are exchange-symmetric (triplet states) and one state that is exchange-antisymmetric (singlet state).

\section{Long-Lived States: Defeating the Foe}

As discussed above, the dipole-dipole coupling, which generates most of the relaxation, is symmetric under exchange. It may be shown that a symmetric relaxation mechanism cannot change an antisymmetric state (the singlet state) into a symmetric state (one of the triplet states). Hence, if the spin system is somehow placed into a singlet state, it will be protected against dipole-dipole relaxation, since there is no other antisymmetric state. The singlet state of a spin-1/2 pair is an example of a long-lived state, which is protected against relaxation by its own symmetry, and the symmetry of the dominant relaxation mechanism.

The protection is not complete, since there are relaxation mechanisms other than dipole-dipole relaxation, and not all of these mechanisms have the same symmetry properties. Nevertheless, protecting a state against dipole-dipole relaxation often extends the lifetime of the state by many factors, and this can be helpful in preserving the lifetime of nuclear hyperpolarization.

It is beyond the scope of this article to describe in detail just how long-lived singlet polarization is generated in practical NMR experiments, and how the lifetime extension effect has been
The singlet state cannot be converted into any of the triplet states by the dipoledipole coupling, since it has the wrong symmetry. It is therefore a long-lived state. 
demonstrated. The effect is real, and was first demonstrated in 2003 on the compound DBT, as sketched above. Very recently, specially-designed molecules have been synthesized which have spectacularly long nuclear singlet lifetimes. In a recent case, the lifetime of nuclear singlet polarization exceeds 1 hour in a roomtemperature solution, even though the spin-lattice relaxation time constant $T_{1}$ of the same system is a few tens of seconds, under identical conditions.

Research is underway, in our group and others, to apply these insights to extending the lifetime of nuclear hyperpolarization, with potential applications to MRI and many other scientific fields [3].

Those interested in the topic may consult the suggested reading to learn more about nuclear spin relaxation and long-lived nuclear singlet states.

Address for Correspondence Malcolm $\mathrm{H}$ Levitt University of Southampton School of Chemistry Southampton, SO17 1BJ, UK. Email:mhl@soton.ac.uk

\section{Suggested Reading}

[1] M H Levitt, Spin Dynamics, Wiley, Chichester (UK), 2nd edition, 2007.

[2] J Kowalewski and L Mäler, Nuclear Spin Relaxation in Liquids: Theory, Experiments and Applications, CRC Press, Boca Raton, Florida, 2006.

[3] M H Levitt, Singlet nuclear magnetic resonance, Annual Review of Physical Chemistry, Vol.63, pp.89-105, 2012. 